\section{Relação entre a retenção de peso nos primeiros três meses pós-parto com ganho de peso e ingestão alimentar durante a gestação}

\section{The relation between weight retention in the first three months post-partum and weight gain and diet during gestation}

Cristina Carra Forte 1

Juliana Rombaldi Bernardi 2

Marcelo Zubaran Goldani 3 Vera Lúcia Bosa 4

\begin{abstract}
Objectives: to study weight retention in women in the first three months post-partum and its correlation with gestational weight gain $(G W G)$ and diet.

Methods: a cohort study of 61 women was conducted. The Food Intake Frequency Questionnaire (FIFQ), International Physical Activity Questionnaire (IPAQ) and a questionnaire on breastfeeding and anthropometric data were applied. Evaluations were conducted between 24 and 48 hours post-partum, and after 7, 15, 30 and 90 days. Weight retention was calculated by subtracting pre-gestational weight from weight three months post-partum. The statistical tests used were the Pearson and Spearman correlations, Student's $t$ test, multiple comparisons with Bonferroni's adjustment, and linear regression.

Results: the mean age was $28 \pm 7.0$ years, with median daily food intake during pregnancy of 3,670.3 $\mathrm{kcal}, \mathrm{GWG}$ of $12.0 \mathrm{~kg}$ and weight retention in the first three months of $3.2 \mathrm{Kg}$. A significant association was found between weight retention in the first three months post-partum and $G W G(p<0.001)$ and parity $(p<0.05)$. For each kilo gained during pregnancy, 0.8 $\mathrm{kg}$ was retained in the first three months.

Conclusions: weight retention three months postpartum was found to be greater the greater the GWG and the number of previous births.

Key words Weight gain, Food consumption, Pregnancy, Postpartum period
\end{abstract}

1,4 Departamento de Nutrição. Universidade Federal do Rio Grande do Sul. Rua Ramiro Barcelos, 2400. Porto Alegre, RS, Brasil. CEP: 90.035-003.E-mail: criscarraforte@yahoo.com.br

2 Núcleo de Estudos em Saúde da Criança e Adolescente. Hospital de Clínicas de Porto Alegre. Universidade Federal do Rio Grande do Sul - NESCA/HCPA/UFRGS. Porto Alegre, RS, Brasil.

3 Departamento de Pediatria. Faculdade de Medicina. Universidade Federal do Rio Grande do Sul. Porto Alegre, RS, Brasil.

\section{Resumo}

Objetivos: estudar a retenção de peso em mulheres nos primeiros três meses pós-parto e sua correlação com ganho de peso gestacional (GPG) $e$ consumo alimentar.

Métodos: estudo de coorte com 61 mulheres. Aplicou-se: Questionário de Frequência Alimentar (QFA), Questionário Internacional de Atividade Física (IPAQ) e questionário referente à amamentação e dados antropométricos. Realizaram-se avaliações entre 24 e 48 horas pós-parto, 7, 15, 30 e 90 dias. A retenção de peso deu-se pela subtração entre peso aos três meses pós-parto e peso pré-gestacional. Os testes estatísticos usados foram: correlações de Pearson e Spearman, teste $t$ de Student, de comparação múltipla com ajuste de Bonferroni $e$ regressão linear.

Resultados: a média de idade foi 28土7,0 anos, com medianas: de consumo alimentar diário na gestação de $3.670,3 \mathrm{kcal}, \mathrm{GPG}$ de $12,0 \mathrm{~kg}$ e retenção de peso nos primeiros três meses de $3,2 \mathrm{Kg}$. Observou-se associação significativa entre retenção de peso nos primeiros três meses pós-parto e o GPG $(p<0,001)$ e a paridade $(p<0,05)$. Para cada quilo ganho durante a gestação $0,8 \mathrm{~kg}$ foi retido nos primeiros três meses.

Conclusões: a retenção de peso três meses pósparto mostrou-se maior quanto maior o GPG durante a gestação e a paridade.

Palavras-chave Ganho de peso, Consumo alimentar, Gestação, Período pós-parto 


\section{Introdução}

A obesidade é considerada um problema emergente de saúde pública, sendo mais prevalente em mulheres. ${ }^{1}$ A retenção de peso após o parto é considerada umas das causas que levam as mulheres com idade fértil a desenvolver obesidade. Esta é influenciada por uma complexa rede de inter-relações, principalmente nos primeiros meses pós-parto, como ganho de peso gestacional, paridade, amamentação, a readaptação do organismo sem o corpo gravídico, a involução uterina, o retorno dos órgãos reprodutivos, o aumento das mamas, a quantidade de líquido corpóreo e o estilo de vida. ${ }^{2,3}$ Além disso, a inadequação do estado nutricional pré-gestacional contribui para o desenvolvimento de diversas intercorrências durante a gestação e no período pós-parto para a nutriz e seu bebê.3-5

O ganho de peso gestacional tem sido descrito como um dos mais importantes fatores determinantes para a retenção de peso pós-parto. ${ }^{6}$ Rebelo et al. 2 observaram que o ganho de peso gestacional total e o índice de massa corporal (IMC) pré-gestacional mantiveram associação significativa com a retenção de peso pós-parto e, juntos, explicam $40,7 \%$ da variabilidade do desfecho no grupo estudado.

No período gestacional ocorrem diversas adaptações fisiológicas, metabólicas e nutricionais no organismo materno. ${ }^{7}$ Essas alterações levam ao aumento das necessidades energéticas, principalmente durante a etapa embrionária em que ocorre o crescimento inicial do feto. ${ }^{3}$ A relação entre o maior consumo de calorias e o ganho de peso elevado na gestação está documentada na literatura. Crispim et al.,8 concluíram que o desequilíbrio da ingestão alimentar durante o período gestacional pode implicar no ganho de peso durante a gravidez. Atualmente, estudos buscam relacionar as características e padrões alimentares durante o período gestacional com o ganho de peso nesse período e com a retenção de peso pós-parto. ${ }^{9,10}$ Cabral, $11 \mathrm{em}$ um estudo com 250 puérperas, observou que o consumo alimentar excessivo pode acarretar maior ganho de peso durante a gestação, e resultar em maior retenção de peso pós-parto.

Considerando a importância do adequado ganho de peso gestacional e a alta prevalência de obesidade após o parto, o presente estudo teve como objetivo avaliar a retenção de peso em mulheres nos primeiros três meses pós-parto e sua correlação com ganho de peso, consumo alimentar e atividade física no período gestacional, além do aleitamento materno.

\section{Métodos}

Trata-se de um estudo de coorte, parte de um estudo maior prospectivo controlado intitulado "Impacto das Variações do Ambiente Perinatal sobre a Saúde do Recém-Nascido nos Primeiros Seis Meses de Vida" (IVAPSA) com início em setembro de 2011 que tem por objetivo principal descrever o impacto das variações no ambiente perinatal nos primeiros seis meses de vida do recém-nascido. $12 \mathrm{O}$ recrutamento das participantes ocorreu no Hospital de Clínicas de Porto Alegre (HCPA) e no Grupo Hospitalar Conceição (GHC). A amostra foi selecionada de maneira consecutiva.

Para este estudo o cálculo do tamanho da amostra foi realizado no programa WinPepi, versão 11.15 , com base no estudo de Rebelo, 2 o qual investigou fatores associados à retenção de peso como idade, escolaridade, IMC pré-gestacional, adequação do ganho de peso total, amamentação exclusiva, paridade, entre outras aos 55 dias pós-parto. Para o cálculo estatístico foi utilizado poder estatístico de $80 \%$, nível de significância de 5\% e uma correlação de 0,4 entre o ganho de peso gestacional total e retenção de peso. Assim, chegou-se ao tamanho amostral de, no mínimo, 47 mulheres, que acrescido de $10 \%$ de perdas, totalizou 52 participantes.

Foram incluídas no estudo puérperas atendidas nos hospitais citados anteriormente e residentes na cidade de Porto Alegre, RS. Os critérios de exclusão foram: mulheres com doenças crônicas como diabetes e hipertensão arterial sistêmica, tabagistas, soropositivas, com idade gestacional inferior a 37, gravidez gemelar ou que seu recém-nascido (RN) apresentasse doenças crônicas, congênitas ou que necessitasse de internação hospitalar e com restrição de crescimento intrauterino (RCIU).

Os entrevistadores, após submetidos a treinamento a fim de uniformizar a coleta de dados, verificavam os nascimentos ocorridos nos Hospitais e as características das puérperas por meio de análise de prontuários hospitalares para posterior inclusão no estudo. As entrevistas com as participantes ocorreram em seis etapas, sendo a primeira no alojamento dos Hospitais (HCPA e GHC), dentro do período de 24 a 48 horas pós-parto. Os demais acompanhamentos foram realizados aos sete, $15,30,90 \mathrm{e}$ 180 dias, sendo os primeiros dois atendimentos e o quarto realizados a domicílio, e o terceiro e último no Centro de Pesquisa Clínica do HCPA. Destaca-se que para o presente trabalho utilizaram-se os dados das entrevistas inicial, aos sete e aos 90 dias.

$\mathrm{Na}$ primeira entrevista, as puérperas foram convidadas a participar do estudo, sendo explicados 
os objetivos do projeto e entregue o termo de consentimento livre e esclarecido (TCLE). Com a aceitação da puérpera, aplicou-se um questionário geral de coleta de dados, do qual foram obtidas informações sobre dados socioeconômicos e demográficos, como idade materna, cor/raça, escolaridade, renda e estado civil. Juntamente, foi aplicado o Questionário Internacional de Atividade Física (IPAQ) referente às atividades do período gestacional o qual foi relatado pelas puérperas. $\mathrm{O}$ instrumento inclui perguntas variadas, como atividades no trabalho, por lazer, esportes ou na própria casa ou jardim. A classificação foi realizada de acordo com a quantidade de vezes na semana de atividade praticada e o tempo total que ocorreram; após foram definidas em sedentárias, irregularmente ativa, ativa e muito ativa. Para o estudo, denominouse puérperas Não Ativas as classificadas como sedentárias e irregularmente ativas e, em Ativas as participantes ativas e muito ativas. 13 Os dados antropométricos como peso pré-gestacional (último peso referido pela puérpera antes de engravidar) e peso antes do parto (na internação hospitalar para o parto) foram coletados da carteira da gestante e/ou do prontuário hospitalar. O ganho de peso gestacional foi obtido pela diferença entre o peso antes do parto e o peso pré-gestacional. O estado nutricional pré-gestacional assim como a recomendação de ganho de peso foi classificado de acordo com o Institute of Medicine (IOM), 142013.

$\mathrm{Na}$ entrevista seguinte, sete dias pós-parto, foram avaliados aspectos referentes ao consumo alimentar durante a gestação. Esta foi realizada em visita domiciliar pré-agendada pelos pesquisadores. $\mathrm{Na}$ visita domiciliar aos três meses pós-parto foram realizadas medidas antropométricas da puérpera e aplicado questionário sobre a amamentação exclusiva que para fins de análise considerou-se consumo de leite materno sem a oferta de outros alimentos, chás ou água. Além disso, avaliou-se a retenção de peso pós-parto (diferença entre o peso nos três meses pósparto e o peso pré-gestacional). A aferição das medidas antropométricas seguiram as recomendações da Organização Mundial da Saúde e Sistema de Vigilância Alimentar e Nutricional (SISVAN), 2011.15

O consumo alimentar durante a gestação foi mensurado por meio de um Questionário de Frequência Alimentar (QFA) semi-quantitativo, adaptado de outro previamente validado para gestantes. ${ }^{16}$ A lista de alimentos do QFA original continha 88 itens alimentares, no qual houve o acréscimo de 15 itens afim de, melhor representar o consumo habitual no período gestacional. As porções correspondentes às medidas caseiras préestabelecida no QFA, foram padronizadas segundo a tabela de Avaliação de Consumo Alimentar em Medidas Caseiras. ${ }^{17}$ Os alimentos não contemplados na tabela foram pesados ou medidos em balança digital, a fim de se obter um valor padrão. As frequências de consumo dos alimentos foram convertidas em equivalentes diários, cujos valores atribuídos foram: "mais de três vezes/dia" $=3$; "duas a três vezes/dia" $=2$; "uma vez/dia" $=1$; "cinco a seis vezes/semana" - $[(5+6) / 2] / 7=0,79$; "duas a quatro vezes/semana" - $[(2+4) / 2] / 7=0,43$; "uma vez/semana" - $1 / 7=0,14$; "uma a três vezes/mês" $[(1+3) / 2] / 30=0,07 ;$ "nunca/quase nunca" $=0$. O cálculo do consumo alimentar foi realizado por meio da Base de Dados de Nutrientes do Departamento de Agricultura dos Estados Unidos (USDA). 18

O presente estudo foi aprovado pelos Comitês de Ética em Pesquisa do GHC e HCPA, com os números de protocolos 11-027 e 11-0097, respectivamente. As puérperas assinaram o Termo de Consentimento Livre e Esclarecido (TCLE).

Cada puérpera obteve seu número de identificação específico, mantendo-se dessa forma, o anonimato dos participantes. Houve revisão dos questionários pelos supervisores do projeto, e os dados foram digitados e processados no programa estatístico SPSS (Statistical Package for the Social Sciences) versão 18.0 (SPSS Inc., Chicago, IL, EUA $\left.^{\circledR}\right)$, com dupla digitação, com posterior conferência e validação dos dados.

Posteriormente, as variáveis contínuas foram expressas como média \pm desvio-padrão (DP) ou por mediana e intervalo interquartil (percentil 25- $\mathrm{P}_{25} \mathrm{e}$ percentil 75- $\mathrm{P}_{75}$ ) e categóricas através de frequências absolutas e relativas. Para avaliar a correlação da retenção de peso e as variáveis de distribuição normal (peso) foi realizada a correlação de Pearson e para as variáveis de distribuição assimétrica (consumo alimentar) foi realizada a correlação de Spearman. O teste $t$ de Student foi realizado para avaliar e comparar as médias de retenção de peso e o aleitamento materno exclusivo aos três meses (sim ou não), paridade (primíparas ou multíparas) e atividade física durante a gestação (ativa e não ativa). Foi realizada regressão linear simples para verificar a retenção de peso para cada quilograma ganho. A variação de peso foi avaliada por meio do teste de comparações múltiplas com ajuste de Bonferroni. Para todas as análises, foi considerado nível de significância de $p<0,05$ e intervalo de confiança de $95 \%$. 


\section{Resultados}

A amostra estudada foi constituída de 61 puérperas, com idade média de $28( \pm 7,07)$ anos, e $55(90,2 \%)$ eram casadas ou moravam com o companheiro. Quanto aos valores de IMC, a maioria $35(57,4 \%)$ das puérperas apresentaram IMC pré-gestacional adequado e, 26 (42,7\%) encontravam-se com excesso de peso (sobrepeso + obesidade). A mediana do consumo alimentar diário relatado durante o período gestacional foi de 3670,3 calorias $\left(\mathrm{P}_{25}\right.$ : 3069,6 kcal; $\left.\mathrm{P}_{75}: 5305,2 \mathrm{kcal}\right)$ e o ganho de peso neste período obteve mediana de $12,0 \mathrm{~kg}\left(\mathrm{P}_{25}: 8.5\right.$ $\mathrm{kg} ; \mathrm{P}_{75}: 16,3 \mathrm{~kg}$ ). A retenção de peso nos primeiros três meses teve mediana de $3,2 \mathrm{~kg}\left(\mathrm{P}_{25}: 0,2 \mathrm{Kg} ; \mathrm{P}_{75}\right.$ : $6.5 \mathrm{Kg}$ ). As características gerais estão descritas na Tabela 1.

Observou-se que a perda de peso maior após o parto ocorreu nos primeiros 15 dias, permanecendo até os três meses, porém com menor intensidade $(p<0,001)$ (Figura 1). A perda de peso após 15 dias pós-parto não foi estatisticamente significante quando realizadas as comparações múltiplas (ajuste de Bonferroni).

Foi observada correlação positiva entre a retenção de peso nos primeiros três meses pós-parto com o ganho de peso gestacional total $(\mathrm{r}=0,796$; $p<0,001$ ), conforme mostrado na Tabela 2 , indicando que cerca de $63 \%$ da variação de retenção de peso aos três meses após o parto pode ser explicado pelo ganho de peso gestacional $\left(\mathrm{r}^{2}=0,63\right)$. Em relação à paridade, foi possível observar que as puérperas multíparas apresentaram retenção de peso maior quando comparadas as primíparas $(p=0,039)$. A retenção de peso foi maior em mulheres que apresentaram maior ganho de peso gestacional $(7,7 \mathrm{~kg} \pm 6,3$; $p<0,001)$, em comparação as mulheres com ganho de peso gestacional adequado $(3,16 \mathrm{~kg} \pm 3 ; p<0,001)$ ou

Tabela 1

Características demográficas, socioeconômicas e nutricionais da amostra (Coorte IVAPSA, Porto Alegre).

\begin{tabular}{|c|c|}
\hline Variáveis & $\mathrm{N}=61$ \\
\hline \multicolumn{2}{|l|}{ Cor da pele/Raça, n (\%) } \\
\hline Branca & $35(57,4)$ \\
\hline Não Branca & $26(42,6)$ \\
\hline Idade (anos), $\bar{X} \pm D P$ & $28,0 \pm 7,0$ \\
\hline Escolaridade (anos), $\bar{X} \pm \mathrm{DP}$ & $9,5 \pm 2,9$ \\
\hline Renda familiar mensal $(R \$), \bar{X} \pm D P$ & $1.913,0 \pm 1.058,2$ \\
\hline \multicolumn{2}{|l|}{ Paridade, $\mathrm{n}(\%)$} \\
\hline Primíparas & $25(41,0)$ \\
\hline Multíparas & $36(59,0)$ \\
\hline \multicolumn{2}{|l|}{ Mora com companheiro, n (\%) } \\
\hline Sim & $55(90,2)$ \\
\hline Não & $6(9,8)$ \\
\hline \multicolumn{2}{|l|}{ Variáveis antropométricas } \\
\hline IMC pré-gestacional adequado n (\%) & $35(57,4)$ \\
\hline sobrepeso n (\%) & $17(27,9)$ \\
\hline obesidade n (\%) & $9(14,8)$ \\
\hline Ganho de peso $(\mathrm{kg})$ gestacional; mediana $\left(\mathrm{P}_{25}-\mathrm{P}_{75}\right)$ & $12,0(8,5-16,3)$ \\
\hline Ganho de peso gestacional insuficiente, n (\%) & $17(27,9 \mathrm{~kg})$ \\
\hline adequado, n (\%) & $22(36,1 \mathrm{~kg})$ \\
\hline excessivo, n (\%) & $22(36,1 \mathrm{~kg})$ \\
\hline Retenção de peso $(\mathrm{kg})$ aos três meses; mediana $\left(P_{25}-P_{75}\right)$ & $3,2(0,2-6,5)$ \\
\hline \multicolumn{2}{|l|}{ Consumo alimentar diário } \\
\hline Calorias totais (kcal); mediana $\left(\mathrm{P}_{25}-\mathrm{P}_{75}\right)$ & $3670,3(3069,6-5305,2)$ \\
\hline Carboidratos (\%VET); mediana $\left(\mathrm{P}_{25}-\mathrm{P}_{75}\right)$ & $61,9(56,0-65,2)$ \\
\hline Proteínas (\%VET); mediana $\left(\mathrm{P}_{25}-\mathrm{P}_{75}\right)$ & $14,1(12,1-17,0)$ \\
\hline Lipídeos (\%VET); mediana $\left(\mathrm{P}_{25}-\mathrm{P}_{75}\right)$ & $26,8(23,3-30,6)$ \\
\hline
\end{tabular}

IVAPSA = Impacto das Variações do Ambiente Perinatal sobre a Saúde do Recém-Nascido nos Primeiros Seis Meses de Vida; $\mathrm{DP}=$ desvio-padrão; IMC= índice de massa corporal $(\mathrm{kg} / \mathrm{m} 2) ; \mathrm{Kcal}=$ calorias. 
insuficiente $(-1,95 \mathrm{~kg} \pm 6,9 ; p<0,001)$ (dados não apresentados). Além disso, foi observado que para cada quilo ganho durante a gestação, cerca de $0,8 \mathrm{~kg}$ foi retido nos primeiros três meses $(\beta=0,794)$ (Figura 2).

No presente estudo não foi observada correlação significativa entre retenção de peso nos três primeiros meses pós-parto e consumo alimentar durante a gestação. Da mesma forma, não foi observada associação significativa entre o desfecho estudado e o aleitamento materno exclusivo ou atividade física.

Variação absoluta do peso das mulheres durante a gestação e nos três primeiros meses após o parto (Coorte IVAPSA, Porto Alegre).

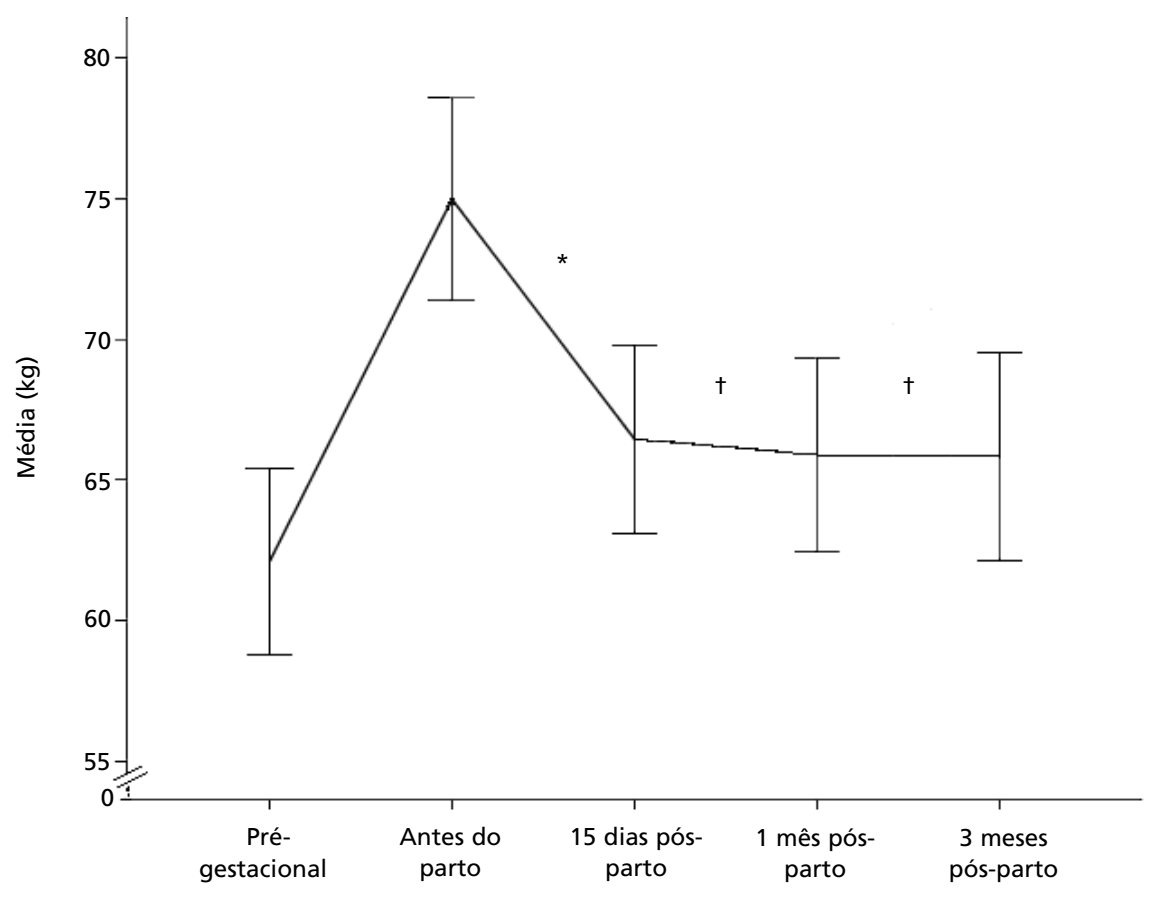

IVAPSA = Impacto das Variações do Ambiente Perinatal sobre a Saúde do Recém-Nascido nos Primeiros Seis Meses de Vida.

Dados apresentados como média e DP; Teste de comparação múltipla com ajuste de Bonferroni para todos os intervalos; ${ }^{*} p<0,01$ em todos os intervalos quando comparados ao peso antes do parto e $t p=1,0$ para intervalos 15 dias pós-parto. 
Tabela 2

Fatores associados à retenção de peso aos três meses (Coorte IVAPSA, Porto Alegre).

\begin{tabular}{|c|c|c|c|}
\hline Variáveis $(n=61)$ & Correlação (r) & Médias & $p$ \\
\hline Ganho de peso $¥(\mathrm{~kg})$ & 0,796 & - & $<0,001^{*}$ \\
\hline \multicolumn{4}{|l|}{ Consumo alimentar $\dagger$} \\
\hline Calorias & $-0,350$ & - & 0,788 \\
\hline Carboidratos (g) & 0,061 & - & 0,643 \\
\hline Proteínas (g) & $-0,013$ & - & 0,920 \\
\hline Lipídeos (g) & 0,020 & - & 0,881 \\
\hline Ácidos graxos saturados (g) & 0,026 & - & 0,844 \\
\hline Ácidos graxos monoinsaturados (g) & 0,002 & - & 0,989 \\
\hline \multirow[t]{2}{*}{ Ácidos graxos poliinsaturados (g) } & 0,041 & - & 0,754 \\
\hline & & $\overline{\mathrm{x}} \pm \mathrm{DP}$ & \\
\hline \multicolumn{4}{|l|}{ Aleitamento materno\# } \\
\hline Exclusivo & & $3,14 \pm 6,64$ & \\
\hline Não exclusivo & & $3,68 \pm 6,92$ & 0,759 \\
\hline \multicolumn{4}{|l|}{ Paridade\# } \\
\hline Multíparas & & $4,84 \pm 5,91$ & \\
\hline Primíparas & & $1,26 \pm 7,32$ & $0,039 *$ \\
\hline \multicolumn{4}{|l|}{ Atividade física\# } \\
\hline Ativa & & $2,49 \pm 7,14$ & \\
\hline Não ativa & & $4,83 \pm 5,78$ & 0,191 \\
\hline
\end{tabular}

‡ Correlação de Pearson; † Correlação de Spearman; * $p<0,05$; \# Teste $t$.

Figura 2

Relação entre ganho de peso gestacional total e retenção de peso aos três meses pós-parto (Coorte IVAPSA, Porto Alegre).

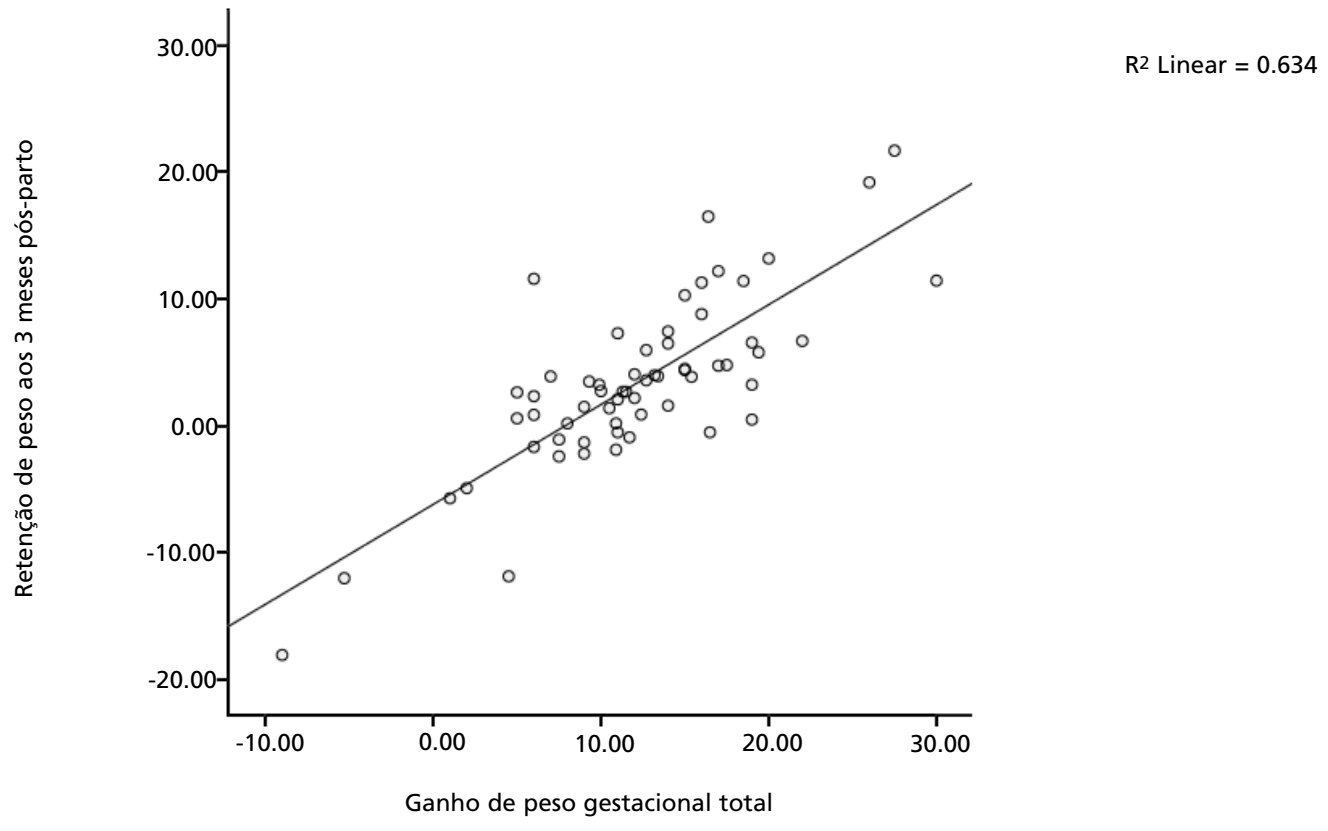

IVAPSA= Impacto das Variações do Ambiente Perinatal sobre a Saúde do Recém-Nascido nos Primeiros Seis Meses de Vida.

Reta de regressão; Equação: Retenção = -6,18 + 0,787 x ganho de peso gestacional total; $\beta=0,794 ; r=0,796 ; p<0,001$. 


\section{Discussão}

O acompanhamento do adequado ganho de peso gestacional é ainda um desafio para as equipes de saúde, no atendimento pré-natal. O presente estudo objetivou avaliar a retenção de peso em mulheres nos primeiros três meses pós-parto e sua relação com ganho de peso, consumo alimentar no período gestacional e outras variáveis relacionadas. Assim, demonstrou-se existir correlação entre a retenção de peso nos primeiros três meses pós-parto e o ganho de peso durante a gestação, como era esperado, devido a ser um componente à retenção e, em mulheres que tiveram mais filhos. No entanto, na amostra, não foi observada correlação com o consumo alimentar e outras variáveis investigadas.

Em mulheres em idade reprodutiva, a gestação tem sido um dos fatores para o desenvolvimento da obesidade devido ao ganho de peso excessivo durante a gestação e a retenção pós-parto. A retenção média de peso aos três meses pós-parto, no presente estudo, foi semelhante à coorte realizada por Rebelo et al.,2 no município do Rio de Janeiro, o qual encontraram valor de $3,2 \mathrm{~kg}( \pm 3,7)$, sendo a variação de $0,6-12,1 \mathrm{~kg}$, aos 55 dias após o parto.

O ganho de peso durante o período gestacional é uma dos principais fatores relacionados à retenção de peso pós-parto e, observou-se no estudo de Rebelo et al. 2 que a cada quilo de peso ganho durante este período, cerca de $50 \%$ foi retido no pósparto. Da mesma forma, a revisão sistemática realizada por Lacerda e Leal19 em 2004, observou-se que de 11 estudos avaliados, dez mostraram associação positiva entre ganho de peso gestacional total e retenção de peso pós-parto. Dessa forma, enfatiza-se a importância do cuidado nutricional não apenas no período pré-natal, mas na atenção à saúde da mulher, de forma geral, incluindo os períodos anterior e posterior à gravidez.

Em relação ao consumo alimentar e retenção de peso, Cabral11 observou que o consumo energético excessivo pode acarretar maior ganho de peso na gravidez, e consequentemente, maior retenção de ganho de peso pós-parto. Segundo Martins e Benício10 o consumo de gordura saturada e alimentos processados durante a gestação revelaram aumento significativo na retenção de peso após o parto, principalmente nos primeiros 15 dias. Lacerda e Leal, ${ }^{19}$ em revisão sistemática, observaram que três estudos entre 28 avaliados havia associação positiva entre o consumo alimentar e a retenção de peso pós-parto, o que não foi encontrado no presente estudo.

Em se tratando de paridade e retenção de peso, a revisão realizada por Lacerda e Leal19 em 2004, assim como o presente estudo, revelou associação entre paridade e retenção de peso pós-parto. Os autores observaram que cinco dos oito estudos analisados apresentaram maior retenção de peso pósparto em mulheres com mais de um filho. A retenção foi de 3,09 $\mathrm{kg}, 3,44 \mathrm{~kg}$ e 3,58 $\mathrm{kg}$ para mulheres com a primeira, segunda e terceira gestações, respectivamente.

A retenção de peso pós-parto quando relacionado com o aumento de peso durante a gestação não foi observada associação significativa entre puérperas com IMC pré-gestacional adequado, sobrepeso e obesidade. Entretanto, Sequeira et al.,20 em estudo de coorte histórico realizado com 495 gestantes inscritas em um centro de saúde, destacaram que $35 \%$ das mulheres apresentaram IMC pré-gestacional sobrepeso ou obesidade e, $46 \%$ delas tiveram ganho de peso gestacional superior ao recomendado. Estas obtiveram média de ganho de peso gestacional de $14,5( \pm 6,0) \mathrm{kg}$, com mínimo de $-2 \mathrm{~kg}$ e máximo de $31 \mathrm{~kg}$. Os autores observaram associação estatisticamente significativa entre IMC pré-gestacional e aumento ponderal de peso.

As mulheres ativas demonstraram retenção de peso menor quando comparadas às mulheres sedentárias, embora sem resultados estatisticamente significativos. Os resultados dos estudos de Sampeselle et al.21 também revelaram efeito positivo da atividade física pós-parto. As mulheres mais ativas reteram menos peso $(3,9 \mathrm{~kg})$ do que as menos ativas $(5,1 \mathrm{~kg})$, enfatizando a importância de programas de atividade física. Boardley et al.22 investigaram atividade física pré e pós-parto, em 335 mulheres, estando apenas o grau de atividade física anterior à gestação associado com a retenção de peso no pósparto. Comparação entre maior e menor quartil de atividade física revelaram uma diferença na retenção de peso de quase $1 \mathrm{~kg}$. Entretanto, em concordância com o presente estudo, Ohlin e Rossner ${ }^{23}$ reportaram que nem os hábitos alimentares, nem o grau de atividade física puderam ser determinados como preditores para a retenção de peso. Isso poderia ser explicado por diminuição do gasto energético durante e depois da gestação.

Em uma meta-análise realizada por Amorim et al. 24 concluiu-se que a perda de peso é eficaz quando as mulheres são submetidas a dieta e a atividade física em conjunto. Do mesmo modo, Ostbye et al., $25 \mathrm{em}$ ensaio clínico randomizado (ERC) com 450 participantes obtiveram resultados semelhantes, tendo o grupo de intervenção uma perda de peso pósparto ligeiramente superior ao observada do grupo controle, porém, sem significância estatística. O 
grupo de intervenção recebeu orientações de alimentação saudável e de atividade física.

As limitações do presente estudo estão relacionadas à análise de consumo alimentar durante a gestação, tendo em vista que a aplicação do questionário para avaliar o consumo foi realizada sete dias após o parto, o que poderia dificultar o relato com precisão das quantidades e dos alimentos ingeridos durante esse período. Além disso, outra limitação comumente observada em estudos sobre retenção de peso consiste na utilização de dados de peso pré-gestacional reportados pela própria mulher. Ressalta-se que o QFA é um instrumento validado para avaliar o consumo alimentar quantitativo e qualitativo utilizado em estudos para este fim. O tamanho amostral mostrou-se adequado, pois pode

\section{Referências}

1. IBGE (Instituto Brasileiro de Geografia e Estatística). Análise da disponibilidade domiciliar de alimentos e do estado nutricional no Brasil: pesquisa de orçamentos familiares. 2010.

2. Rebelo F, Castro MBT, Dutra CL, Schilussel MM, Kac G. Fatores associados à retenção de peso pós-parto em uma corte de mulheres, 2005-2007. Rev Bras Saúde Matern Infant. 2010; 2 (10): 219-27.

3. Lucyk JM, Furumoto RV. Necessidades nutricionais e consumo alimentar na gestação: uma revisão. Com Ciência Saúde. 2008; 19 (4): 353-63.

4. Padilha PC, Salinders C, Machado RCM, Silva CL, Bull A, Sally EOF, Accioly E. Associação entre o estado nutricional pré-gestacional e a predição do risco de intercorrências gestacionais. Rev Bras Ginecol Obstet. 2007; 29 (10): 5118

5. Fazio ES, Nomura RMY, Dias MCG, Sugaib M. Consumo dietético de gestantes e ganho ponderal materno após aconselhamento nutricional. Rev Bras Ginecol Obstet. 2011; 33 (2): 87-92.

6. Kac G. Fatores determinantes da retenção de peso no pósparto: uma revisão da literatura. Cad Saúde Pública. 2001; 17 (3): 455-66

7. Freitas ES, Bosco SMD, Sippel CA, Lazzaretti RK Recomendações nutricionais na gestação. Rev Destaque Acadêmico (Univates). 2010; 2 (3): 81-95.

8. Crispim SP, Silva MMS, Ribeiro RCL. Validação de questionários de freqüência alimentar. Nutrição Brasil. 2003; 2 (5): 286-290.

9. Uusitalo U, Arkkola T, Ovaskainen M-L, Kronberg-Kippila C, Kenward MG, Veijola R. Unhealthy dietary patterns are associated with weight gain during pregnancy among Finnish women. Public Health Nutr. 2009; 12 (12): 2392-9.

10. Martins APB, Benício MHA. Influência do consumo alimentar na gestação sobre a retenção de peso pós-parto. Rev Saúde Pública. 2011; 5 (45): 870-7.

11. Cabral MMLGF. Consumo de proteína na gestação e retenção de peso no pós-parto [trabalho de investigação] detectar uma correlação de até 0,4 nos desfechos estudados como, por exemplo, entre o ganho de peso gestacional e a retenção de peso.

A retenção de peso aos três meses pós-parto apresentou correlação positiva com o ganho de peso durante a gestação, porém não houve diferença quanto ao consumo alimentar durante esse período. Além disso, as mulheres com maior número de filhos apresentam maior retenção de peso aos três meses pós-parto. Remete-se a necessidade de futuros estudos prospectivos em diferentes populações de gestantes e puérperas a fim de esclarecer a influência de fatores nutricionais como alimentação, estado nutricional e atividade física durante a gestação em relação à retenção de peso pós-parto.

Porto: Faculdade de Ciências da Nutrição e Alimentação, Universidade do Porto; 2010.

12. Bernardi JR, Ferreira CF, Nunes M, Silva CH, Bosa VL, Silveira PP, Goldani MZ. Impact of perinatal different intrauterine environments on child growth and development in the first six months of life - IVAPSA birth cohort: rationale, design, and methods. BMC Pregnancy and Childbirth. 2012; 12 (25): 1-11.

13. International Physical Activity Questionnaire. Downloadable questionnaires. [acesso em 3 jun 2013]. Disponivel em: www.portalagita.org.br/uploads/agita_ saopaulo/arquivo/IPAQ_classificacao.pdf

14. IOM (Institute of Medicine). Weight gain during pregnancy: reexamining the guidelines. Washington, DC: The National Academies Press; 2013.

15. Brasil. Ministério da Saúde. Secretaria de Atenção à Saúde. Departamento de Atenção Básica. Orientações para coleta e análise de dados antropométricos em serviços de saúde: Norma Técnica do Sistema de Vigilância Alimentar e Nutricional - SISVAN. Brasília, DF; 2011. (Série G. Estatística e Informação em Saúde).

16. Giacomello A, Schimidt MI, Nunes MAA, Ducan BB, Soares RM, Manzolli P. Validação relativa de Questionário de Frequência Alimentar em gestantes usuárias de serviços do Sistema Único de Saúde em dois municípios no Rio Grande do Sul. Rev Bras Saúde Matern Infant. 2008; 8 (4): 445-54.

17. Pinheiro ABV, Lacerda EMA, Benzecry EH, Gomes MCS, Costa VM. Tabela para avaliação do consumo alimentar em medidas caseiras. São Paulo: Atheneu; 2005.

18. USDA (National Nutrient Database for Standard Reference). In Release 25, U.S. Department of Agriculture, Agricultural Research Service. 2013.

19. Lacerda EMA, Leal MC. Fatores associados a retenção e o ganho de peso pós-parto: uma revisão sistemática. Rev Bras Epidemiol. 2004; 7 (2): 187-200.

20. Sequeira J, Simões C, Colaço V, Dias J. Evolução ponderal na gravidez, preditores e consequências: estudo retrospectivo. Rev Port Med Geral Fam. 2013; 29: 98-104. 
21. Sampselle CM, Seng J, Yeo S, Killion C, Oakley D. Physical activity and postpartum well-being. J Obstet Gynecol Neonatal Nurs. 1999; 28: 41-9.

22. Boardley DL, Sargent RG, Coker AL, Hussey JR, Sharpe PA. The relation-ship between diet, activity and other factors, and postpartum weight change by race. Obstet Ginecol. 1995; 86: 834-8.

23. Ohlin A, Rossner S. Trends in eating patterns, physical activity and socio-demographics factors in relation to postpartum body weight development. Brit J Nutr. 1994; 71: $457-70$
24. Amorim AR, Linne YM, Lourenco PM. Diet or exercise, or both, for weight reduction in women after childbirth. Cochrane Database Systematic Reviews (3), CD005627; 2007.

25. Ostbye T, Krause KM, Lovelady CA, Morey MC, Bastian LA, Peterson BL, Swamy GK, Brouwer RJ, McBride CM. Active mothers postpartum: a randomized controlled weight-loss intervention trial. Am J Prev Med. 2009; 37 (3): 173-80.

Recebido em 18 de setembro de 2014

Versão final apresentada em 1 de junho de 2015

Aprovado em 1 de julho de 2015 